\title{
Elliptical orbits in the phase-space quantization
}

\author{
Órbitas elíticas na quantização do espaço de fase
}

\author{
Leonardo Andreta de Castro, Carlos Alexandre Brasil, Reginaldo de Jesus Napolitano \\ Instituto de Física de São Carlos, Universidade de São Paulo, São Carlos, SP, Brasil
}

Recebido em 21 de março de 2016. Aceito em 22 de maio de 2016

\begin{abstract}
The energy levels of hydrogen-like atoms are obtained from the phase-space quantization, one of the pillars of the old quantum theory, by three different methods - (i) direct integration, (ii) Sommerfeld's original method, and (iii) complex integration. The difficulties come from the imposition of elliptical orbits to the electron, resulting in a variable radial component of the linear momentum. Details of the calculation, which constitute a recurrent gap in textbooks that deal with phase-space quantization, are shown in depth in an accessible fashion for students of introductory quantum mechanics courses.

Keywords: old quantum mechanics, phase-space quantization.

Os níveis de energia de átomos hidrogenoides são obtidos através da quantização do espaço de fase, um dos pilares da teoria quântica velha, por três métodos diferentes - (i) integração direta, (ii) o método original de Sommerfeld, e (iii) integração complexa. As dificuldades surgem da imposição de órbitas elíticas ao elétron, resultando numa componente radial variável do momentum linear. Minúcias do cálculo, que constituem uma omissão comum nos livros-textos que lidam com a quantização do espaço de fase, são mostrados em detalhe de uma maneira acessível a estudantes de cursos de mecânica quântica introdutória.
\end{abstract}

Palavras-chave: antiga mecânica quântica, quantização do espaço de fase.

\section{Introduction}

Few expressions are more frustrating to the student than "it is easy to prove that" followed by a nontrivial result. On the one hand, when well employed, that is, when the steps necessary to reach the final result are a natural consequence of what was explained before, the expression improves the reader's self-confidence by stimulating the use of creativity to fill the logical and mathematical gaps in finding the solution. On the other hand, its inadequate use can make the student lose too much time in a fruitless pursuit of misguided paths, leaving them eventually discouraged when a simple sentence or set of references could point them to the right direction.

This article addresses the specific but recurrent inadequate usage of "it is easy to show that" in many quantum mechanics textbooks $[1,6]$ when deriving the energy levels of a hydrogen-like atom. [6]

\footnotetext{
*Endereço de correspondência: leoadec@gmail.com.
}

The problem consists in solving an integral resulting from the phase-space quantization conditions $6 \begin{array}{ll}6 & 11\end{array}$ - fundamental in the old quantum theory - when the electronic orbits are allowed to be elliptical (as opposed to circular), thus allowing for non-zero radial component of the momentum.

Specifically, if the electron orbits the nucleus in an elliptical trajectory, with the radius varying between $r_{\max }$ and $r_{\text {min }}$, with constant energy $E$ and angular momentum $L$, the quantization of the radial action will be given by the integral

$$
2 \int_{r_{\min }}^{r_{\max }} \mathrm{d} r \sqrt{-\frac{L^{2}}{r^{2}}+\frac{2 Z m e^{2}}{r}+2 m E}=n_{r} h
$$

where $h$ is Planck's constant, $m$ is the mass of the electron, $-e$ its charge, and $Z$ the number of protons in the nucleus. $n_{r}$ is a positive integer, the radial 
quantum number. Our aim is to solve this to find an expression for the energy $E$.

Introductory quantum mechanics textbooks that deal with the old quantum theory and present Eq. (1) omit the necessary steps to solve it - or provide general guidelines based on properties of ellipses - and then present the final result of the energy. For example, Eisberg and Resnick's books [1,2] only show the quantization conditions for the angular and linear momenta, and then simply give a final expression relating the angular momentum and the axes of the ellipsis to the energy levels. Tomonaga, $[3]$ by his turn, employs the expression "the integration in Eq. (20.8) [equivalent to our Eq. (1)] is elementary and gives" before reproducing the final result. Bohm [4] leaves the integral as an exercise, and Born [5] only cites the result to highlight the remarkable coincidence between this and Dirac's later results. He later solves the equation in an appendix using the same method Sommerfeld 6, 10, employed to solve the integral.

This fact does not constitute a severe hindrance in the learning process, since Schrödinger's (and Dirac's) equation provides the complete solutions for the hydrogen atom. [12,13] However, the hydrogen atom is one of the most important elementary systems that can be solved with quantum mechanics (together with the potential well and the harmonic oscillator), and we consider relevant that the students know how to work out the details even in the old quantum theory. Here, we explore these calculations in detail, so the readers can focus more of their time on the physical interpretation of the results.

The article is organized as follows: Sec. 2 provides a brief historical introduction to the old quantum theory; Sec. 3 shows how the integral that quantizes the radial component of the action can be computed without previous knowledge of its orbits; Sec. 4 describes the original method Arnold Sommerfeld employed to solve the integral using the properties of an ellipse and a few other tricks. Conclusions and further historical context are presented in Sec. 5. Appendices present details of the more difficult integrations.

\section{History Overview}

In the period of time between Max Planck's proposed quantization of energy in 1900 [14 15] and the development of the first forms of matrix [16] and wave 17 mechanics - beginning in 1925 and 1926, respectively - the physics of the atomic phenomena evolved into a theory now known as old quantum mechanics (OQM). 15, 18, Analyses of Planck's reasoning to derive the quantum of action $h$ are beyond the scope of this article and can be found in the references, 14, 15, 19,21 along with translations of Planck's original works. 22 24 Below, we will see how this was a hybrid theory that prescribed a classical approach to the problem before the quantization could be, somehow, introduced.

\subsection{Niels Bohr's contributions}

The greatest achievement of the OQM was the explanation of the discrete emission spectrum of hydrogen, a problem that had been intriguing physicists as early as the 19th century. 15 The first step was Niels Bohr's atomic model, 25 31] which allowed the theoretical derivation of Balmer's formula, 32, 33 and which was shortly after complemented by Arnold Sommerfeld's work $10,11,34,35$ that explained the fine structure - first observed by Albert Michelson in 1891. 15

A common oversight in introductory quantum mechanics textbooks [1-3 is to introduce Bohr's atomic model by just citing his postulates, as if they were simple and obvious considerations, when in fact these were the result of a careful analysis of the spectral data and of the theoretical toolkit available at the time. 29 The very quantization of the linear momentum is commonly presented as a postulate, despite it actually being a consequence.

Bohr presented his atomic model in three articles published in the Philosophical Magazine in 1913, 25 28 "On the constitution of atoms and molecules." In the first one, he presents two postulates, reproduced literally below:

1. "That the dynamical equilibrium of the systems in the stationary states can be discussed by help of the ordinary mechanics, while the passing of the systems between different stationary states cannot be treated on that basis.

2. "That the latter process is followed by the emission of a homogeneous radiation, for which the relation between the frequency and the amount of energy emitted is the one given by Planck's theory."

Initially, Bohr, aware that the circular orbits are unstable, makes a classical energetic analysis of the 
electron-proton system - i. e., he applies postulate (1). This postulate introduces the stationary states and was responsible for a large share of the criticism towards his model. 36 Bohr then assumes that Balmer's empirical formula could be explained in terms of a difference in energies, which he associates to different orbits. The mechanical frequency of rotation of the electron around the nucleus and the frequency of emitted radiation are associated with one another and with Planck's constant - here, postulate (2) is applied. The distinction between frequencies is an innovative aspect of his model. 36,37 Bohr's solution offered the following expression for the energies:

$$
E_{n}=-\frac{R_{\mathrm{H}}}{n^{2}},
$$

where $n$ is an integer, the principal quantum number, which specifies a given circular orbit of the electron, and $R_{\mathrm{H}}$ is the Rydberg constant of the hydrogen atom.

The greatest achievement of Bohr's model was to derive Balmer's formula and $R_{\mathrm{H}}$ in terms of fundamental constants:

$$
R_{\mathrm{H}}=\frac{1}{4 \pi} \frac{m e^{4}}{\hbar^{3}}
$$

where there appeared as well a parameter that would later become of widespread use, Bohr's radius $r_{1}$ the radius of the orbit that remains closest to the nucleus:

$$
r_{1}=\frac{\hbar^{2}}{m e^{2}} .
$$

In 1922, Niels Bohr was awarded a Nobel prize in Physics "for his services in the investigation of the structure of atoms and of the radiation emanating from them". 38

In his analysis of the hydrogen atom, [25, 28] to find the aforementioned relation between the rotation and emission frequencies, 29] Bohr employs still before formalizing it - one of the forms of the correspondence principle. [15, 39,40] This principle [39] (which states that when we take the limits of some basic parameters of quantum mechanics we must find the classical results again), despite being vital in OQM, has different formulations due to Planck and Bohr (and also by Heisenberg, but we will not approach this here), which are not equivalent [41]. In short, according to Planck, the correspondence principle consisted in the recovery of the classical result when $\hbar \rightarrow 0$, [42] while to Bohr it corresponds to taking $n \rightarrow \infty$, where $n$ is a quantum number related to the frequencies of the system. Afterwards, Bohr 39 would provide a more precise formulation of his principle:

"As far as the frequencies are concerned we see that in the limit where $n$ is large, there exists a close relation between the ordinary theory [classical picture] of radiation and the theory of spectra based on [the two quantum postulates]."

In fact, to be universally valid, the principle of correspondence requires both formulations, with the limits $n \rightarrow \infty$ and $h \rightarrow 0$ linked by the action $J=n h .43$ One can treat Bohr's atom emphasizing Bohr's formulation of the uncertainty principle (related to $r \rightarrow \infty$ ), instead of using directly the balance of the Coulomb force and the centripetal force in the electron. 44

In 1916, while attempting to quantize the hydrogen atom subject to external electric fields (Stark effect) or magnetic fields (Zeeman effect), Sommerfeld noticed that Bohr's rules led to a greater number of spectral lines than observed experimentally. With the help of the correspondence principle, Bohr could derive selection rules, which specified how quantum numbers can vary; and, with Hendrik Kramers's assistance, he explained the intensity of the lines in the hydrogen spectrum. 37]

Correcting the calculation for the reduced mass of the system with Kramers, 37 Bohr managed to successfully explain the spectrum of helium, increasing the acceptance of his theory. 15 Also remarkable are the spectra of a few molecules, 27, 28 which agree with experimental data. 30,31

\subsection{Arnold Sommerfeld's formulation}

OQM was based on the following rules: 15

- the use of classical mechanics to determine the possible motions of the system;

- the imposition of certain quantum conditions to select the actual or allowed motions;

- the treatment of radiative processes as transitions between allowed motions subject to Bohr's frequency formula.

During calculation - and not just to verify the consistency of the final solutions - an adequate version of the correspondence principle could be used. 
Analyses of the phase space were already being conducted by Max Planck, [15,20,45 after all Planck's constant 14,21,24 $h$ has dimension of action (and angular momentum). Planck's proposal was the rule defined by

$$
\iint \mathrm{d} q \mathrm{~d} p=h
$$

where $q$ is a generalized coordinate, and $p$ its corresponding conjugate momentum. 46 Planck discussed this subject in the first Solvay Congress, 15, 20 while Sommerfeld, in the same event, presented a postulate according to which "in 'every purely molecular process', the quantity of action

$$
\int_{0}^{\tau} L \mathrm{~d} t=\hbar
$$

is exchanged, where $\tau$ is the duration of the process and $L$ is the Lagrangian." 20 This elicited a discussion with his colleagues, including Einstein. Finally, Sommerfeld presented to the Bavarian Academy of Sciences the phase-space quantization in two communications from December, 1915, [34] and January, 1916. [35] In the same year he would write a treatise for the Anallen der Physik [47] where he detailed his ideas about the quantization of each degree of freedom for atomic processes, 10,11, 18 so that, for $f$ degrees of freedom, we have $f$ equations

$$
\oint \mathrm{d} q_{k} p_{k}=n_{k} h,
$$

where $q_{k}$ is a generalized coordinate and $p_{k}$ is its corresponding momentum, $n_{k}$ is a non-negative integer, $k$ varies between 1 and $f$, and the integral is extended through a whole period of $q_{k}$. 3, 15

Other three authors have proposed Eq. (3): (i) Jun Ishiwara in 1915; [9] (ii) William Wilson in 1916; [7] (iii) Niels Bohr in 1918. [48] Here we will focus on Sommerfeld's contributions, but an overview of these versions can be found in the references. [8, 15

As mentioned above, Bohr's model treated the atom as having many possible circular electronic orbits around the nucleus, designated by the principal quantum number $n$, which, for the values $1,2,3$ and 4, corresponded in spectroscopic notation to $s$, $p, d$ and $f, 49$ from Single, Principal, Diffuse and Fundamental - this notation was used by Sommerfeld, among others. [15, 50

Sommerfeld, on the other hand, sought a more abstract interpretation for the numerical regularities in the spectral lines, a less pictorial and more formal approach that was even called "number mysticism." 50 51] His colleague Wilhelm Wien in Munich called Sommerfeld's Atomistik an Atom-Mystik. 50 This point of view was synthesized by Pauli: 51,52

"At that time there were two approaches to the difficult problems connected with the quantum of action. One was an effort to bring abstract order to the new ideas by looking for a key to translate classical mechanics and electrodynamics into quantum language which would form a logical generalization of these. This was the direction which was taken by Bohr's 'correspondence principle.' Sommerfeld, however, preferred, in view of the difficulties which blocked the use of the concepts of kinematical models, a direct interpretation, as independent of models as possible, of the laws of spectra in terms of integral numbers, following, as Kepler once did in his investigation of the planetary system, an inner feeling for harmony. Both methods, which did not appear to me irreconcilable, influenced me. The series of whole numbers $2,8,18,32 \ldots$ giving the lengths of the periods in the natural system of chemical elements, was zealously discussed in Munich, including the remark of the Swedish physicist, Rydberg, that these numbers are of the simple form $2 n^{2}$, if $n$ takes on all integer values. Sommerfeld tried especially to connect the number 8 and the number of corners of a cube." [italics ours]

The highlighted sentences contain the essence of the method employed by Sommerfeld and his disciples, which he would apply as well to the Zeeman Effect. 45 51] The analogy with Kepler led Sommerfeld to consider the general case of elliptical orbits (in this non-literal sense). [18] We will see these calculations in detail in the next section.

\section{Quantization of the Orbits}

The description of phenomena in the OQM was heavily based on their classical dynamics. For this reason, the atom would be studied through the knowledge of classical celestial mechanics, a field that had already 
collected many results for a point particle subject to a central force proportional to the inverse square distance. The Lagrangian formalism is especially useful for future quantization of the action:

$$
\mathcal{L}=K-V=\frac{m \dot{\mathbf{r}}^{2}}{2}+\frac{Z e^{2}}{r},
$$

where the electron is at position $\mathbf{r}$, has mass $m$ and charge $-e$, and is orbiting $Z$ protons at the origin whose mass is approximated as infinite. 53 . $K$ and $V$ represent kinetic and potential energy. [54

The equation of motion for any given coordinate $q$ in this formulation is:

$$
\frac{\mathrm{d}}{\mathrm{d} t} \frac{\partial \mathcal{L}}{\partial \dot{q}}=\frac{\partial \mathcal{L}}{\partial q} .
$$

For the Cartesian coordinate $z$ and the Lagrangian (4), we have:

$$
m \ddot{z}=-\frac{Z e^{2}}{r^{3}} z .
$$

If we choose $x y$ plane as the one that contains both the initial position $\mathbf{r}(0)$ and velocity vector $\dot{\mathbf{r}}(0)$, we will have $z(0)=\dot{z}(0)=0$, and as a consequence $\ddot{z}=\dot{z}=z=0$ for every instant of time. For this reason, the orbit can be described in two dimensions, and we just need to quantize these two coordinates according to Eq. (3) to find the energy levels of the atom:

$$
\begin{aligned}
& \oint \mathrm{d} q_{1} p_{1}=n_{1} h, \\
& \oint \mathrm{d} q_{2} p_{2}=n_{2} h .
\end{aligned}
$$

In accordance to the Lagrangian formalism, $p_{1}, p_{2}$ are the partial derivatives of the Lagrangian with respect to the first time derivative of these coordinates.

We may choose these two coordinates in any way that seems us fit, as long as you know how the generalized momenta depend on the generalized coordinates, so you can proceed to calculate the integrals.

Fortunately, solving the equations of motion may prove unnecessary for our purposes, which are just of finding the energy levels of the atom. We may take a shortcut by using the conservation of total energy $E$ to establish a relationship between the generalized coordinates and the generalized momenta:

$$
E=\frac{m \dot{\mathbf{r}}^{2}}{2}-\frac{Z e^{2}}{r} .
$$

This $E$ must be negative for orbits, otherwise the electron would not be bound to the nucleus, and free to escape towards infinity.

From Eq. (5), one can find $p_{1}$ as a function of $p_{2}, q_{1}, q_{2}$. An additional relation may be necessary to solve the integrals, which may come from a clever manipulation of the equations of motion:

$$
\begin{aligned}
& \dot{p}_{1}=\frac{\partial \mathcal{L}}{\partial q_{1}}, \\
& \dot{p}_{2}=\frac{\partial \mathcal{L}}{\partial q_{2}} .
\end{aligned}
$$

The most traditional method of solution would employ the polar coordinates $r, \theta$. In these coordinates, the Lagrangian (4) becomes:

$$
\mathcal{L}=\frac{m \dot{r}^{2}}{2}+\frac{m r^{2} \dot{\theta}^{2}}{2}+\frac{Z e^{2}}{r},
$$

yielding the generalized momenta:

$$
\begin{aligned}
& p_{r}=\frac{\partial \mathcal{L}}{\partial \dot{r}}=m \dot{r}, \\
& p_{\theta}=\frac{\partial \mathcal{L}}{\partial \dot{\theta}}=m r^{2} \dot{\theta} .
\end{aligned}
$$

The angular momentum, henceforth represented by $L$, is a constant, because the Lagrangian does not depend on $\theta$ :

$$
\frac{\mathrm{d}}{\mathrm{d} t} L=\frac{\partial \mathcal{L}}{\partial \theta}=0 .
$$

As in a complete orbit the angle $\theta$ goes from 0 to $2 \pi$, the phase-space quantization condition translates as:

$$
\oint \mathrm{d} \theta p_{\theta}=\int_{0}^{2 \pi} \mathrm{d} \theta L=n_{\theta} h,
$$

or $L=n_{\theta} \hbar$. Fortunately, this also means that the total energy in Eq. (5) can be written just in terms of $p_{r}$ and $r$ :

$$
E=\frac{p_{r}^{2}}{2 m}+\frac{L^{2}}{2 m r^{2}}-\frac{Z e^{2}}{r} .
$$

Solving for $p_{r}$, this momentum can be written as a function of a single variable:

$$
p_{r}(r)= \pm \sqrt{-\frac{L^{2}}{r^{2}}+\frac{2 Z m e^{2}}{r}+2 m E} .
$$

Revista Brasileira de Ensino de Física, vol. 38, nº 3, e3318, 2016 
The sign is chosen according to whether $r$ is increasing (positive) or decreasing (negative). The points where $p_{r}=0$ are the extremities of the movement in $r$. They correspond to the roots of the following second-degree polynomial:

$$
L^{2}\left(\frac{1}{r}\right)^{2}-2 Z m e^{2}\left(\frac{1}{r}\right)-2 m E=0 .
$$

Solving it, we find the values of the radius in the periapsis (closest distance to the nucleus) and apoapsis (farthest distance to the nucleus):

$$
\left\{\begin{array}{l}
r_{\min }=\frac{1}{\frac{Z m e^{2}}{L^{2}}+\frac{1}{L} \sqrt{2 m E+\frac{Z^{2} m^{2} e^{4}}{L^{2}}}}, \\
r_{\max }=\frac{1}{\frac{Z m e^{2}}{L^{2}}-\frac{1}{L} \sqrt{2 m E+\frac{Z^{2} m^{2} e^{4}}{L^{2}}}} .
\end{array}\right.
$$

These two extremes become the same when $E=$ $-Z^{2} m e^{4} / 2\left(n_{\theta} \hbar\right)^{2}$, which is the case of circular orbits. The energy levels of an elliptical orbit, however, will also depend on the quantum number of the radius, $n_{r}$, and its relation with energy can only be found by solving the integral (equivalent to Eq. 1):

$$
\begin{aligned}
& \oint \mathrm{d} r p_{r} \\
& =2 \int_{r_{\min }}^{r_{\max }} \mathrm{d} r \sqrt{-L^{2}\left(\frac{1}{r}-\frac{1}{r_{\max }}\right)\left(\frac{1}{r}-\frac{1}{r_{\min }}\right)} \\
& =n_{r} h,
\end{aligned}
$$

where we used the fact that the integral through the entire orbit corresponds to two equal terms from $r_{\min }$ to $r_{\max }$, and then back from $r_{\max }$ to $r_{\min }$, which we simplified to a single integral multiplied by a factor 2. The sign chosen is positive because in this region $r$ is increasing.

This integral can be calculated in the complex plane with the help of the residue theorem, which is explained in Appendix A. Here, we will adopt an approach that requires simpler calculus by simply changing variables to a $\vartheta$ such that:

$$
r^{-1}=\left(\frac{r_{\min }^{-1}+r_{\max }^{-1}}{2}\right)+\left(\frac{r_{\min }^{-1}-r_{\max }^{-1}}{2}\right) \sin \vartheta .
$$

Clearly, $\vartheta=\pi / 2$ when $r=r_{\min }$, and $\vartheta=-\pi / 2$ when $r=r_{\text {max }}$. With a little algebra the integral becomes the following:

$$
2 L \int_{-\pi / 2}^{\pi / 2} \mathrm{~d} \vartheta \frac{\cos ^{2} \vartheta}{(\varepsilon+\sin \vartheta)^{2}}=n_{r} h .
$$

where we defined

$$
\varepsilon \equiv \frac{r_{\min }^{-1}+r_{\max }^{-1}}{r_{\min }^{-1}-r_{\max }^{-1}}=\frac{1}{\sqrt{1+2 m E\left(\frac{L}{Z m e^{2}}\right)^{2}}}>1 .
$$

The integral from Eq. (9) is found in mathematical handbooks, and a direct method of solving it that any student of quantum mechanics can understand can be found in Appendix B. The final result is

$$
\int_{-\pi / 2}^{\pi / 2} \mathrm{~d} \vartheta \frac{\cos ^{2} \vartheta}{(\varepsilon+\sin \vartheta)^{2}}=\pi \frac{\varepsilon}{\sqrt{\left(\varepsilon^{2}-1\right)}}-\pi .
$$

Hence, back to Eq. (9), we find, at last:

$$
2 \pi L\left\{\frac{1}{\sqrt{1-\varepsilon^{-2}}}-1\right\}=n_{r} h .
$$

From the definition of $\varepsilon$ in Eq. 10 ,

$$
\frac{1}{\sqrt{1-\varepsilon^{-2}}}=\frac{1}{\sqrt{-2 m E}} \frac{Z m e^{2}}{L} .
$$

Knowing this, we can solve Eq. (11) for $E$ to find the energy levels:

$$
E=-\frac{Z^{2} m e^{4}}{2\left(n_{r} \hbar+L\right)^{2}}
$$

Equivalently, we can replace the angular momentum $L$ by its quantized values $n_{\theta} \hbar: 4$

$$
E=-\frac{m}{2 \hbar^{2}} \frac{Z^{2} e^{4}}{\left(n_{r}+n_{\theta}\right)^{2}}
$$

These are the energy levels of the elliptical orbits, which are the same as in the case of circular orbits, except that now the energy levels are degenerate, because there are two quantum numbers in the denominator. This also indicates that this result - in which we arrived using only the integrals, and no further information about the form of the trajectory - is correct. Using properties of the ellipse, however, these procedures can be made a little shorter, as seen in the next section.

\section{A Few Shortcuts}

In the following two subsections, we will see how to derive the same answer from Eq. 12 using the parametric formula of the ellipse:

$$
r(\theta)=a \frac{1-\varepsilon^{2}}{1+\varepsilon \cos \theta},
$$


where $a>0$ is the semi-major axis, and $\varepsilon$ is a number confined to the interval $0<\varepsilon<1$ called eccentricity. Note that this definition of $\varepsilon$ is different from the one used in the previous section, but the use of the same Greek letter is useful due to the similar role both play in the integrand.

Inspecting Eq. (13), we can see that its extrema occur when $r=a(1 \pm \varepsilon)$, which correspond to the $r_{\min }$ and $r_{\max }$ from Eq. (7):

$$
\left\{\begin{array}{l}
r_{\min }=a(1-\varepsilon)=\frac{1}{\frac{Z m e^{2}}{L^{2}}+\frac{1}{L} \sqrt{2 m E+\frac{Z^{2} m^{2} e^{4}}{L^{2}}}}, \\
r_{\max }=a(1+\varepsilon)=\frac{1}{\frac{Z m e^{2}}{L^{2}}-\frac{1}{L} \sqrt{2 m E+\frac{Z^{2} m^{2} e^{4}}{L^{2}}}} .
\end{array}\right.
$$

Adding or multiplying these two, we find out that:

$$
\begin{cases}r_{\min } r_{\max }= & a^{2}\left(1-\varepsilon^{2}\right)=-\frac{L^{2}}{2 m E} \\ \frac{r_{\min }+r_{\max }}{2}= & a=-\frac{Z e^{2}}{2 E}\end{cases}
$$

This pair of results may prove useful below.

\subsection{Integrating in theta}

The solution of Eq. (1), as performed in the previous section, despite being proposed as an exercise in certain textbooks, 4 is not how Sommerfeld quantized the atom in his original 1916 article. 6 He used the fact that the radial momentum $p_{r}$ will inevitably be a function of the angle $\theta$ to integrate in the other coordinate instead:

$$
\oint \mathrm{d} r p_{r}=\int_{0}^{2 \pi} \mathrm{d} \theta \frac{\mathrm{dr}}{\mathrm{d} \theta} p_{r}(\theta)=n_{r} h .
$$

Invoking the fact that $L=m r^{2} \dot{\theta}$ is a constant of motion, we can write the whole integrand in terms of $r$ :

$$
p_{r}(\theta)=m \frac{\mathrm{d} r}{\mathrm{~d} t}=m \frac{\mathrm{d} r}{\mathrm{~d} \theta} \dot{\theta}=\frac{L}{r^{2}} \frac{\mathrm{d} r}{\mathrm{~d} \theta} .
$$

Hence, we only need $r$ as a function of $\theta$ to calculate:

$$
L \int_{0}^{2 \pi} \mathrm{d} \theta\left(\frac{1}{r} \frac{\mathrm{d} r}{\mathrm{~d} \theta}\right)^{2}=n_{r} h
$$

This at first may look like an increase in the difficulty of the problem, because it will require writing $r$ and $p_{r}$ in terms of $\theta$. But if we know Eq. (13), the derivative we need is just:

$$
\frac{1}{r} \frac{\mathrm{d} r}{\mathrm{~d} \theta}=\frac{\varepsilon \sin \theta}{1+\varepsilon \cos \theta} .
$$

Therefore, the integral in Eq. 15 can be simplified to

$$
L \int_{0}^{2 \pi} \mathrm{d} \theta \frac{\sin ^{2} \theta}{\left(\varepsilon^{-1}+\cos \theta\right)^{2}}=n_{r} h .
$$

This trigonometric integral is very similar to Eq. (9) from the last section, and a practical way of solving it can be found in Appendix B. In short, the final result is:

$$
\int_{0}^{2 \pi} \mathrm{d} \theta \frac{\sin ^{2} \theta}{\left(\varepsilon^{-1}+\cos \phi\right)^{2}}=2 \pi\left\{\frac{1}{\sqrt{\left(1-\varepsilon^{2}\right)}}-1\right\},
$$

or, in Eq. 16),

$$
1-\varepsilon^{2}=\frac{L^{2}}{\left(n_{r} \hbar+L\right)^{2}} .
$$

How do we relate $\left(1-\varepsilon^{2}\right)$ to our known constants? From Eqs. (14), we know that

$$
\left(1-\varepsilon^{2}\right)=-\left(-\frac{2 E}{Z e^{2}}\right)^{2} \frac{L^{2}}{2 m E}=-\frac{2 E L^{2}}{Z^{2} m e^{4}}
$$

so that Eq. (17) yields the final answer we had found in the previous section by direct integration:

$$
E=-\frac{Z^{2} m e^{4}}{2\left(n_{r} \hbar+L\right)^{2}}
$$

As you can see, both methods are very much analogous. The main difference is that Sommerfeld's original approach requires a little more geometrical insights.

\subsection{Integrating in time}

Another shortcut to the final solution employs the virial theorem. 40 We begin, once again, from the quantization of the action

$$
\begin{aligned}
& \oint \mathrm{d} r p_{r}=\oint \mathrm{d} r m \dot{r}=n_{r} h, \\
& \oint \mathrm{d} \theta L=\oint \mathrm{d} \theta m r^{2} \dot{\theta}=n_{\theta} h .
\end{aligned}
$$

This time, however, instead of solving the integrals separately, we change variables to time and sum the contributions:

$$
\int_{0}^{T} \mathrm{~d} t m\left(\dot{r}^{2}+r^{2} \dot{\theta}^{2}\right)=h\left(n_{r}+n_{\theta}\right),
$$

where $T$ is the orbital period. 
Now divide both sides by $2 T$. From Eq. (6), we know that the left-hand side became the time average of the kinetic energy of the system, which according to the virial theorem, is equal to the average total energy with sign changed.

Hence, the total energy of the electron in this elliptical orbit, which is a constant, will be:

$E=-\frac{1}{2 T} \int_{0}^{T} \mathrm{~d} t m\left(\dot{r}^{2}+r^{2} \dot{\theta}^{2}\right)=-\frac{1}{2 T} h\left(n_{r}+n_{\theta}\right)$.

Now, we just have to establish a relationship between the period $T$ and other known parameters. To do this, we invoke conservation of linear momentum (or Kepler's second law), which states that $L \mathrm{~d} t=m r^{2} \mathrm{~d} \theta$. Integrating over the whole orbit, the area integral becomes the total area of the ellipse, $\pi a^{2} \sqrt{1-\varepsilon^{2}}$ :

$$
\frac{1}{2} L T=m \pi a^{2} \sqrt{1-\varepsilon^{2}} .
$$

From Eqs. 14, we can rewrite $a$ and $1-\varepsilon^{2}$ in terms of other constants:

$$
a^{2} \sqrt{1-\varepsilon^{2}}=\left(-\frac{Z e^{2}}{2 E}\right) \sqrt{-\frac{L^{2}}{2 m E}}=-\frac{Z e^{2}}{2 E} \frac{L}{\sqrt{-2 m E}},
$$

thus yielding

$$
\frac{1}{2 T}=\frac{E}{2 \pi} \frac{\sqrt{-2 m E}}{Z m e^{2}} .
$$

Returning to Eq. (18), we find

$$
E=-\frac{Z^{2} m e^{4}}{2 \hbar^{2}\left(n_{r}+n_{\theta}\right)^{2}},
$$

which are once again the energy levels we were looking for.

\section{Further Developments}

As seen above, Sommerfeld quantized separately the linear and angular momenta, associating them with the quantum numbers $n_{r}$ and $n_{\theta}$. This results again in Bohr's formula, Eq. (2), but this time

$$
n=n_{r}+n_{\theta} .
$$

The value $n=0$ is excluded $a d$ hoc for being unphysical. 18.
In Sec. 3 we observed that different combinations of $n_{r}$ and $n_{\theta}$ can result in the same $n$, which defines the energy of the orbit. Therefore, Sommerfeld's model introduces degenerate states for the energy and reaches Bohr's 1913 result through an alternative path. However, there still lingered the problem of fine structure, which required one further step.

Attempting to find an expression that included these lines as well, Sommerfeld proposed a threedimensional quantization 15,18 that eliminated the confinement of orbits to a single plane. Moreover, 11] using the relativistic formula for the mass at a given velocity $v$ :

$$
m=\frac{m_{0}}{\sqrt{1-(v / c)^{2}}}
$$

where $m_{0}$ is the rest mass of the electron, Sommerfeld would find a formula that explained the fine structure of the hydrogen spectrum, 15, 18, writing the fine structure constant also in terms of three fundamental constants:

$$
\alpha=\frac{e^{2}}{\hbar c}
$$

and becoming the first to see that this constant could be written as the ratio between the orbital velocity of the electron in the closest orbit and the speed of light. $55-57$

A simple relativistic treatment of Bohr's atom (with circular orbits) results in an energy correction involving terms with $\alpha$, but these values do not differ significantly from the original non-relativistic treatment. [58] Thus Sommerfeld's theory acquired real importance, becoming the highest achievement of OQM, [18] and a confirmation of special relativity. In his Nobel lecture, [59] Planck would speak of it with clear enthusiasm:

"...A. Sommerfeld showed that from a logical extension of the laws of quantum distribution in systems with several degrees of freedom, and out of consideration of the variability of the inertial mass in accordance with the relativity theory, that magic formula [Balmer's series formula] arose before which both the hydrogen and the helium spectrum had to reveal the riddle of their fine structure, to such an extent that the finest presentday measurements, those of F. Paschen, could be explained generally through it 
- an achievement fully comparable with that of the famous discovery of the planet Neptune whose existence and orbit was calculated by Leverrier before the human eye had seen it." [italics ours]

In 1929, Arthur Eddington noticed that the value of $\alpha$ could be written as approximately the inverse of an integer: first $\alpha^{-1} \sim 136$, then $\alpha^{-1} \sim 137$. This numerical aspect and the fact that $\alpha$ can be written as a dimensionless combination of fundamental constants led to an $\alpha$-numerology, a search for deeper meanings for $\alpha$. [55, 56] Curiously, in 1950, the annihilation of a positron by an electron and the resulting emission of two photons was observed with a precision of $137^{-1}$. 60,61 The relativistic treatment for circular orbits sets an upper limit for the atomic number at $Z<\alpha^{-1} \sim 137,[58$ which as of today has not been violated. What would Dr. Eddington have to say about that?

Another success of the OQM, accomplished by Sommerfeld and his assistant Hendrik Kramers, was the initial explanation of the Stark effect. $15,62,63$ In this case, despite the very good agreement with the experimental data available at the time, even Johannes Stark himself showed reservations towards the theory. [57, 63 An important role was fulfilled by Karl Schwarzchild, who made the link between the quantization of the phase integral (3) and the action-variables of the Hamilton-Jacobi theory. 15, 45, 46, 62, 63 This was a natural extension for Schwarzchild, as the action-angle variables and the Hamilton-Jacobi theory are tools much used in celestial mechanics, a field he was most familiar with. 64

We see, then, that the OQM brought advances in many problems, but its gaps would soon be brought to the fore. The theory did not take into account: 18 (i) the spin, necessary for the complete treatment of the total angular momentum of the electron; (ii) the wave nature of particles, necessary to take into account its probabilistic character; 65 67] and (iii) the indistinguishability of particles under certain circumstances. Points (i) and (ii) are directly related to the Pauli exclusion principle. 1, 12, 15] In the case of the Stark effect, 62, 63 for example, the explanation involved ad hoc rules to select the electronic orbits that best fit the experimental data, and the results depended on the system of coordinates on which these conditions were imposed.
How could the result of the fine structure of the spectrum given by the OQM, a heuristic theory with its many gaps, be the same as the one provided by the modern quantum mechanics, after all the contributions of Heisenberg, Schrödinger and Dirac? This is due to the symmetry involved in Sommerfeld's hypothesis of the elliptical orbits and the use of the relativistic mass, which correspond, in modern quantum mechanics, to considering relativistic effects and adding the spin-orbit interaction. 68, 69

Arnold Sommerfeld had great influence also in the consolidation of modern quantum mechanics, and among his students [15] we can mention Isidor Isaac Rabi, Edward Uhler Condon, Peter Debye, Wolfgang Pauli, Werner Heisenberg, Hans Bethe and Alfred Landé, 15, 50, 51,70 the first four laureates of the Nobel prize in Physics. Ironically, Sommerfeld did not receive such an honor.

His curious teaching style was summarized by himself as:

"Personal instruction in the highest sense of the word is best based on intimate personal acquaintanceship. Ski trips with my students offered the best opportunity for that."

His book Atombau und Spektrallinien [6] would be known as "the Bible of quantum spectroscopy" and is said to have impressed David Hilbert, Max Born, and Pieter Zeeman. 50,62

His more formal and abstract approach would end up influencing Bohr himself, who would develop a merely symbolic interpretation of quantum mechanics in which the classical orbits did not represent the actual movement of the electrons. But his ideas went further beyond, and the quantization condition Eq. (3)

$$
\oint \mathrm{d} q p=n h
$$

would influence Heisenberg [37] in deriving the commutation relation for the operators $\hat{q}, \hat{p}$ :

$$
[\hat{q}, \hat{p}]=i \hbar .
$$

Despite being an incomplete theory of strong heuristic character, the OQM allowed for the first application to the atom of the Planck's hypothesis concerning the quantum of light, and allowed an intuitive understanding of the problem. Due to this historical importance, we hope that in clarifying one 
of the aspects of the OQM we have helped not only researchers interested in the early development of quantum mechanics, but also instructors who can use this paper as an instructional aid to assist their students in navigating the possible solutions to this problem.

\section{A. Complex Integration}

Here we will consider how to integrate in the complex plane an integral of the kind found in Eq. 86, 22,71 that is,

$$
\mathscr{I}=2 \int_{r_{\min }}^{r_{\max }} \mathrm{d} r \sqrt{\left(\frac{1}{r_{\min }}-\frac{1}{r}\right)\left(\frac{1}{r}-\frac{1}{r_{\max }}\right)},
$$

which can also be split in two terms as follows:

$$
\begin{aligned}
& \mathscr{I}=\int_{r_{\min }}^{r_{\max }} \mathrm{d} r \sqrt{\left(\frac{1}{r_{\min }}-\frac{1}{r}\right)\left(\frac{1}{r}-\frac{1}{r_{\max }}\right)} \\
& +\int_{r_{\max }}^{r_{\min }} \mathrm{d} r\left[-\sqrt{\left(\frac{1}{r_{\min }}-\frac{1}{r}\right)\left(\frac{1}{r}-\frac{1}{r_{\max }}\right)}\right]
\end{aligned}
$$

In the complex plane, the variable of integration is $z=|z| e^{i \theta}$, with the phase $\theta$ in the interval $[0,2 \pi)$. Then, we can think of the two terms on the righthand side of Eq. 20) as resulting from the complex integral:

$$
\mathscr{I}=\oint_{C} \mathrm{~d} z f(z)
$$

where $C$ is the contour of Fig. 1 in the limit in which $\varepsilon \rightarrow 0^{+}$and $\delta \rightarrow 0^{+}$, with the function $f(z)$ defined in such a way that, in this limit, it tends to

$$
-\sqrt{\left(\frac{1}{r_{\min }}-\frac{1}{r}\right)\left(\frac{1}{r}-\frac{1}{r_{\max }}\right)}
$$

from the first and second quadrants of the complex plane, and it tends to

$$
\sqrt{\left(\frac{1}{r_{\min }}-\frac{1}{r}\right)\left(\frac{1}{r}-\frac{1}{r_{\max }}\right)}
$$

from the third and fourth quadrants.

We can see that $f(z)$ is discontinuous when $z$ crosses the segment $\left[r_{\min }, r_{\max }\right]$, which we will call a "cut line." Nevertheless, $f(z)$ can be proven 72,73 to be continuous everywhere else in the complex plane, except at the origin, where it is singular. The domain of definition of $f(z)$ in the complex plane comprises all the complex numbers except the cut line and zero. In its domain, $f(z)$ is analytical.

Now, consider the contours of Fig. 2. According to the residue theorem, the integral of $f(z)$ along the contour $C^{\prime} \cup(-C)$ is given by

$$
\oint_{C^{\prime} \cup(-C)} \mathrm{d} z f(z)=2 \pi i \operatorname{Res}_{z=0}[f(z)],
$$

where $\operatorname{Res}_{z=0}[f(z)]$ denotes the residue of $f(z)$ at zero. It is easy to see that the integral of Eq. (22) can be expressed as the sum of two other integrals:

$$
\begin{aligned}
& \oint_{C^{\prime} \cup(-C)} \mathrm{d} z f(z)=\oint_{C^{\prime}} \mathrm{d} z f(z) \\
& +\oint_{-C} \mathrm{~d} z f(z)=\oint_{C^{\prime}} \mathrm{d} z f(z)-\oint_{C} \mathrm{~d} z f(z)
\end{aligned}
$$

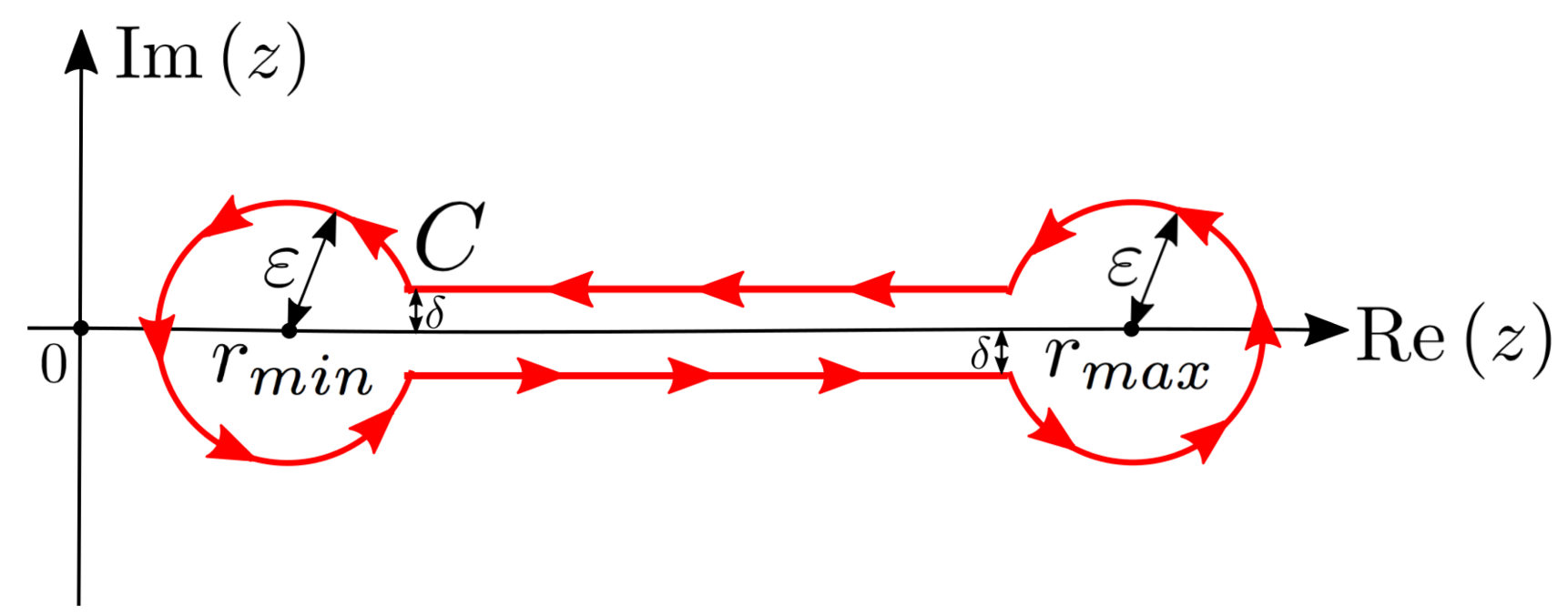

Figure 1: Path of the integration of Eq. (21) in the complex plane. 


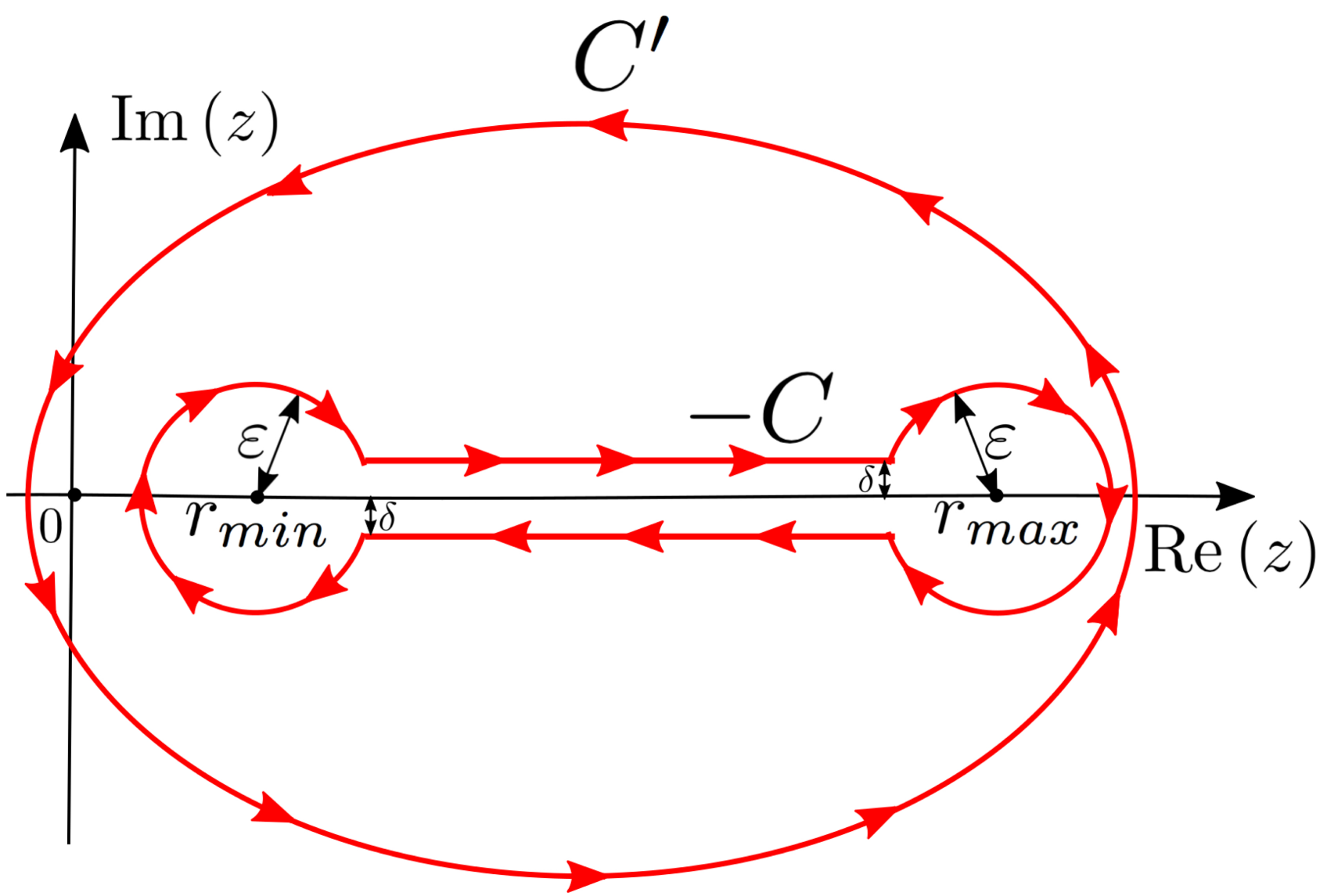

Figure 2: Paths $-C$ and $C^{\prime}$ present in Eq. 22). Here, the contour represented as $-C$ is simply the same contour $C$ from Fig. 1, but traversed in the clockwise direction.

In the limit in which $\varepsilon \rightarrow 0^{+}$and $\delta \rightarrow 0^{+}$, Eqs. 21), (22) and (23) yield

$$
\mathscr{I}=\oint_{C^{\prime}} \mathrm{d} z f(z)-2 \pi i \operatorname{Res}_{z=0}[f(z)]
$$

Thus, if we can calculate the integral of $f(z)$ on the contour $C^{\prime}$, we can find $\mathscr{I}$.

Since $f(z)$ is analytical everywhere outside the region bounded by the contour $C^{\prime}$, it can be expanded in a Laurent series about the origin $f(z)=$ $\sum_{n=-\infty}^{+\infty} a_{n} z^{n}$ where the coefficients are given by:

$$
a_{n}=\frac{1}{2 \pi i} \oint_{C^{\prime}} \mathrm{d} z \frac{f(z)}{z^{n+1}}
$$

for all $n \in \mathbb{Z}$. Integrating both sides of the Laurent series on $C^{\prime}$ gives:

$$
\oint_{C^{\prime}} \mathrm{d} z f(z)=\oint_{C^{\prime}} \mathrm{d} z \sum_{n=-\infty}^{+\infty} a_{n} z^{n}=a_{-1}
$$

since $\oint_{C^{\prime}} \mathrm{d} z z^{n}$ vanishes for all $n \in \mathbb{Z}$, except for $n=-1$. We can also obtain the same result from the series $\sum_{n=-\infty}^{+\infty} a_{n-2} w^{-n}$, namely,

$$
\oint_{C_{0}} \mathrm{~d} w \sum_{n=-\infty}^{+\infty} a_{n-2} w^{-n}=a_{-1},
$$

where $w \in \mathbb{C}$ and $C_{0}$ is a circular contour centered at the origin of the complex plane, in the limit when its radius tends to zero. From Eqs. (26) and (27), we obtain:

$$
\oint_{C^{\prime}} \mathrm{d} z f(z)=\oint_{C_{0}} \mathrm{~d} w \sum_{n=-\infty}^{+\infty} a_{n-2} w^{-n}=\oint_{C_{0}} \mathrm{~d} w \frac{1}{w^{2}} \sum_{n=-\infty}^{+\infty} a_{n} w^{-n}
$$


Noticing the sum on the right-hand side of Eq. 28 is a Laurent series expansion of $f\left(w^{-1}\right)$, we can rewrite it as

$$
\oint_{C^{\prime}} \mathrm{d} z f(z)=\oint_{C_{0}} \mathrm{~d} w \frac{1}{w^{2}} f\left(\frac{1}{w}\right)
$$

It is clear that the function $f(1 / w) / w^{2}$ can only have a singularity at the origin, if any, within the region bounded by the closed curve $C_{0}$. Thus, according to the residue theorem, Eq. (29) gives:

$$
\oint_{C^{\prime}} \mathrm{d} z f(z)=2 \pi i \operatorname{Res}_{w=0}\left[\frac{1}{w^{2}} f\left(\frac{1}{w}\right)\right] .
$$

Usually, one defines the extended complex plane as the usual plane and the point at infinity, [73] such that the residue of Eq. (30) can be rewritten as the residue at infinity of the function $f(z)$ :

$$
\operatorname{Res}_{z=\infty}[f(z)] \equiv \operatorname{Res}_{w=0}\left[-\frac{1}{w^{2}} f\left(\frac{1}{w}\right)\right] .
$$

Hence, substituting Eqs. (30) and (31) into Eq. 24), we obtain:

$$
\mathscr{I}=-2 \pi i \operatorname{Res}_{z=\infty}[f(z)]-2 \pi i \operatorname{Res}_{z=0}[f(z)] .
$$

With the concept of an extended complex plane, we can think of Eq. (32) as simply the integration of $f(z)$ on the contour $-C$, as defined in Fig. 2. It is as if the function $f(z)$ had only two singularities outside of the region bounded by $-C$ : at zero and at infinity. Let us now proceed with the calculation of both residues.

First, let us calculate the residue at zero. For $r \in\left(r_{\min }, r_{\max }\right)$, let us write:

$$
\sqrt{\left(\frac{1}{r_{\min }}-\frac{1}{r}\right)\left(\frac{1}{r}-\frac{1}{r_{\max }}\right)}=\frac{1}{\sqrt{r_{\min } r_{\max }}} \frac{\sqrt{\left(r-r_{\min }\right)\left(r_{\max }-r\right)}}{r} .
$$

Since $f(z)$ is continuous in the neighborhood of the origin, we consider $z$ approaching zero from the first quadrant. With a little algebra it is possible to write:

$$
f(z)=i \exp \left(i \frac{\phi_{\min }+\phi_{\max }}{2}\right) \frac{1}{\sqrt{r_{\min } r_{\max }}} \frac{\sqrt{\left|z-r_{\min }\right|\left|z-r_{\max }\right|}}{z}, \operatorname{Re}(z), \operatorname{Im}(z)>0
$$

where $\phi_{\min }$ and $\phi_{\max }$ are defined as the phases of $z-r_{\min }$ and $z-r_{\max }$, respectively:

$$
\left\{\begin{array}{l}
\left(z-r_{\min }\right)=\left|z-r_{\min }\right| \exp \left(i \phi_{\min }\right) \\
\left(z-r_{\max }\right)=\left|z-r_{\max }\right| \exp \left(i \phi_{\max }\right)
\end{array}\right.
$$

From Eqs. (34), we easily see that, in the limit in which $z$ approaches, from the first quadrant, a point $r$ in the real axis within the interval $\left(r_{\min }, r_{\max }\right)$, then $\theta \rightarrow 0^{+}, \phi_{\min } \rightarrow 0$, and $\phi_{\max } \rightarrow \pi$. This justifies our choice of sign in Eq. (33), because in the first quadrant we defined $f(z)$ as having negative sign:

$$
f(z) \rightarrow \frac{i \exp \left(i \frac{\pi}{2}\right)}{\sqrt{r_{\min } r_{\max }}} \frac{\sqrt{\left|r-r_{\min }\right|\left|r-r_{\max }\right|}}{r}=-\sqrt{\left(\frac{1}{r_{\min }}-\frac{1}{r}\right)\left(\frac{1}{r}-\frac{1}{r_{\max }}\right)} .
$$

From Eq. (33), it follows that

$$
\operatorname{Res}_{z=0}[f(z)]=\lim _{z \rightarrow 0}[z f(z)]=i \exp (i \pi)=-i
$$

since, using Eqs. (34), $\phi_{\min } \rightarrow \pi$ and $\phi_{\max } \rightarrow \pi$ as $z \rightarrow 0$ from the first quadrant. 
Let us use Eq. (33) to calculate the residue at infinity by making $|z| \equiv R \in \mathbb{R}$ approach infinity with $\theta \rightarrow 0^{+}$, that is, keeping $z$ on the real axis. From Eqs. (34) we see that, in this limit, $\phi_{\min }+\phi_{\max } \rightarrow 0$. Hence,

$$
z^{2} f(z)=\frac{i R}{\sqrt{r_{\min } r_{\max }}} \sqrt{\left(R-r_{\min }\right)\left(R-r_{\max }\right)} .
$$

Let $w=1 / z=1 / R$. If $w \rightarrow 0$, then $R \rightarrow \infty$. Eq. (36) then becomes:

$$
\frac{1}{w^{2}} f\left(\frac{1}{w}\right)=\frac{i}{w^{2} \sqrt{r_{\min } r_{\max }}} \sqrt{1-\left(r_{\min }+r_{\max }\right) w+w^{2}}
$$

that is,

$$
\frac{1}{w^{2}} f\left(\frac{1}{w}\right)=\frac{i}{\sqrt{r_{\min } r_{\max }}}\left[\frac{1}{w^{2}}-\frac{1}{2}\left(r_{\min }+r_{\max }\right) \frac{1}{w}+\frac{1}{2}+\ldots\right] .
$$

The residue is the coefficient of $1 / w$, that is, from Eqs. (31) and 37):

$$
\operatorname{Res}_{z=\infty}[f(z)]=\operatorname{Res}_{w=0}\left[-\frac{1}{w^{2}} f\left(\frac{1}{w}\right)\right]=i \frac{\left(r_{\min }+r_{\max }\right)}{2 \sqrt{r_{\min } r_{\max }}}
$$

Hence, substituting Eqs. (35) and (38) into Eq. (32), we obtain:

$$
\mathscr{I}=2 \pi\left[\frac{\left(r_{\min }+r_{\max }\right)}{2 \sqrt{r_{\min } r_{\max }}}-1\right] .
$$

According to the values of the sum and product of $r_{\min }$ and $r_{\max }$ given in Eq. (14), and the definition of $\mathscr{I}$ given in Eq. (19), this is equivalent to stating:

$$
2 \int_{r_{\min }}^{r_{\max }} \mathrm{d} r \sqrt{L^{2}\left(\frac{1}{r_{\min }}-\frac{1}{r}\right)\left(\frac{1}{r}-\frac{1}{r_{\max }}\right)}=L \mathscr{I}=2 \pi L\left[-\frac{Z e^{2}}{2 E} \frac{\sqrt{-2 m E}}{L}-1\right] .
$$

Equating Eq. (39) to $n_{r} h$, as in Eq. (8), we find the same energy levels of Eq. (12):

$$
E=-\frac{Z^{2} m e^{4}}{2\left(n_{r} \hbar+L\right)^{2}}
$$

\section{B. Solving the trigonometric integrals}

In Sec. 3, we have to solve an integral of the form

$$
\int_{-\pi / 2}^{\pi / 2} \mathrm{~d} \vartheta \frac{\cos ^{2} \vartheta}{(\varepsilon+\sin \vartheta)^{2}}
$$

In Sec. 4, we have another integral that looks almost the same as the previous one:

$$
\int_{0}^{2 \pi} \mathrm{d} \theta \frac{\sin ^{2} \theta}{\left(\varepsilon^{-1}+\cos \theta\right)^{2}}
$$

Actually, if we change variables in (40) to $\phi=\pi / 2-\vartheta$, we notice that both are remarkably similar:

$$
\int_{-\pi / 2}^{\pi / 2} \mathrm{~d} \vartheta \frac{\cos ^{2} \vartheta}{(\varepsilon+\sin \vartheta)^{2}}=\int_{0}^{\pi} \mathrm{d} \theta \frac{\sin ^{2} \theta}{(\varepsilon+\cos \theta)^{2}}
$$


We may save some time, therefore, if we solve a more general integral that encapsulates the results of both (40) and (41):

$$
\int_{0}^{N \pi} \mathrm{d} \theta \frac{\sin ^{2} \theta}{(\varepsilon+\cos \theta)^{2}}
$$

where $N>0$ is a positive integer. This is still an integral that requires some effort to be solved. The first step consists in employing integration by parts, which can be used once you notice that

$$
\frac{\partial}{\partial \theta} \frac{1}{(\varepsilon+\cos \theta)}=\frac{\sin \theta}{(\varepsilon+\cos \theta)^{2}} .
$$

Therefore,

$$
\int_{0}^{N \pi} \mathrm{d} \theta \frac{\sin ^{2} \theta}{(\varepsilon+\cos \theta)^{2}}=\int_{0}^{N \pi} \mathrm{d} \theta \sin \theta \frac{\partial}{\partial \theta} \frac{1}{(\theta+\cos \theta)}=-\int_{0}^{N \pi} \mathrm{d} \theta \frac{\cos \theta}{\varepsilon+\cos \theta} .
$$

The remaining integral can be simplified through the simple trick of adding $\varepsilon-(-\varepsilon)$ to the numerator:

$$
-\int_{0}^{N \pi} \mathrm{d} \theta \frac{\cos \theta}{\varepsilon+\cos \theta}=\int_{0}^{N \pi} \mathrm{d} \theta \frac{\varepsilon-(\varepsilon+\cos \theta)}{\varepsilon+\cos \theta}=\int_{0}^{N \pi} \mathrm{d} \theta \frac{\varepsilon}{\varepsilon+\cos \theta}-N \pi .
$$

The cosine function is periodic, so the integrand in the extant integral is the same in every period $[n \pi,(n+1) \pi]$, for every $n$ from 0 to $N-1$. Therefore,

$$
\int_{0}^{N \pi} \mathrm{d} \theta \frac{\varepsilon}{\varepsilon+\cos \theta}=\sum_{n=0}^{N-1} \int_{n}^{(n+1) \pi} \mathrm{d} \theta \frac{\varepsilon}{\varepsilon+\cos \theta}=N \int_{0}^{\pi} \mathrm{d} \theta \frac{\varepsilon}{\varepsilon+\cos \theta} .
$$

Now, we change variables to $w=\tan (\theta / 2)$. This results in the following differential:

$$
\frac{1}{2} \mathrm{~d} \theta=\frac{\mathrm{dw}}{1+w^{2}},
$$

and allows us to express the cosine as follows:

$$
\cos \theta=\cos ^{2}\left(\frac{\theta}{2}\right)-\sin ^{2}\left(\frac{\theta}{2}\right)=\frac{1-\tan ^{2}(\theta / 2)}{1+\tan ^{2}(\theta / 2)}=\frac{1-w^{2}}{1+w^{2}} .
$$

Therefore, the integral in Eq. 45) becomes:

$$
\int_{0}^{\pi} \mathrm{d} \theta \frac{\varepsilon}{\varepsilon+\cos \theta}=2 \int_{0}^{\infty} \mathrm{d} w \frac{\varepsilon}{\varepsilon\left(1+w^{2}\right)+\left(1-w^{2}\right)}=\frac{2 \varepsilon}{\varepsilon-1} \int_{0}^{\infty} \mathrm{d} w \frac{1}{\left(w+w_{0}\right)\left(w-w_{0}\right)},
$$

where $w_{0} \equiv i \sqrt{(\varepsilon+1) /(\varepsilon-1)}$. Note that the number in the square root will always be greater than zero, because $\varepsilon>1$ in both occasions treated in this article (in the second integral, it represents the inverse of the eccentricity, which is always a number between zero and one for an ellipse).

Using partial fractions,

$$
\int_{0}^{\infty} \mathrm{d} w \frac{1}{\left(w-w_{0}\right)\left(w+w_{0}\right)}=\frac{1}{2 w_{0}}\left(\int_{0}^{\infty} \mathrm{d} w \frac{1}{w-w_{0}}-\int_{0}^{\infty} \mathrm{d} w \frac{1}{w+w_{0}}\right)=\frac{1}{2 w_{0}} \int_{-\infty}^{\infty} \mathrm{d} w \frac{1}{w-w_{0}} .
$$

Added to the integral from 0 to $\infty$, we have an integral over the whole real line:

$$
\int_{0}^{\pi} \mathrm{d} \theta \frac{\varepsilon}{\varepsilon+\cos \theta}=\frac{\varepsilon}{\varepsilon-1} \frac{1}{w_{0}} \int_{-\infty}^{\infty} \mathrm{d} w \frac{1}{w-w_{0}} .
$$

What is the value of the integral of $w$ through the real line? We can use Cauchy's residue theorem to perform this integration through a semi-circle of infinite radius that encompasses $w_{0}$ (see Fig. 3): 


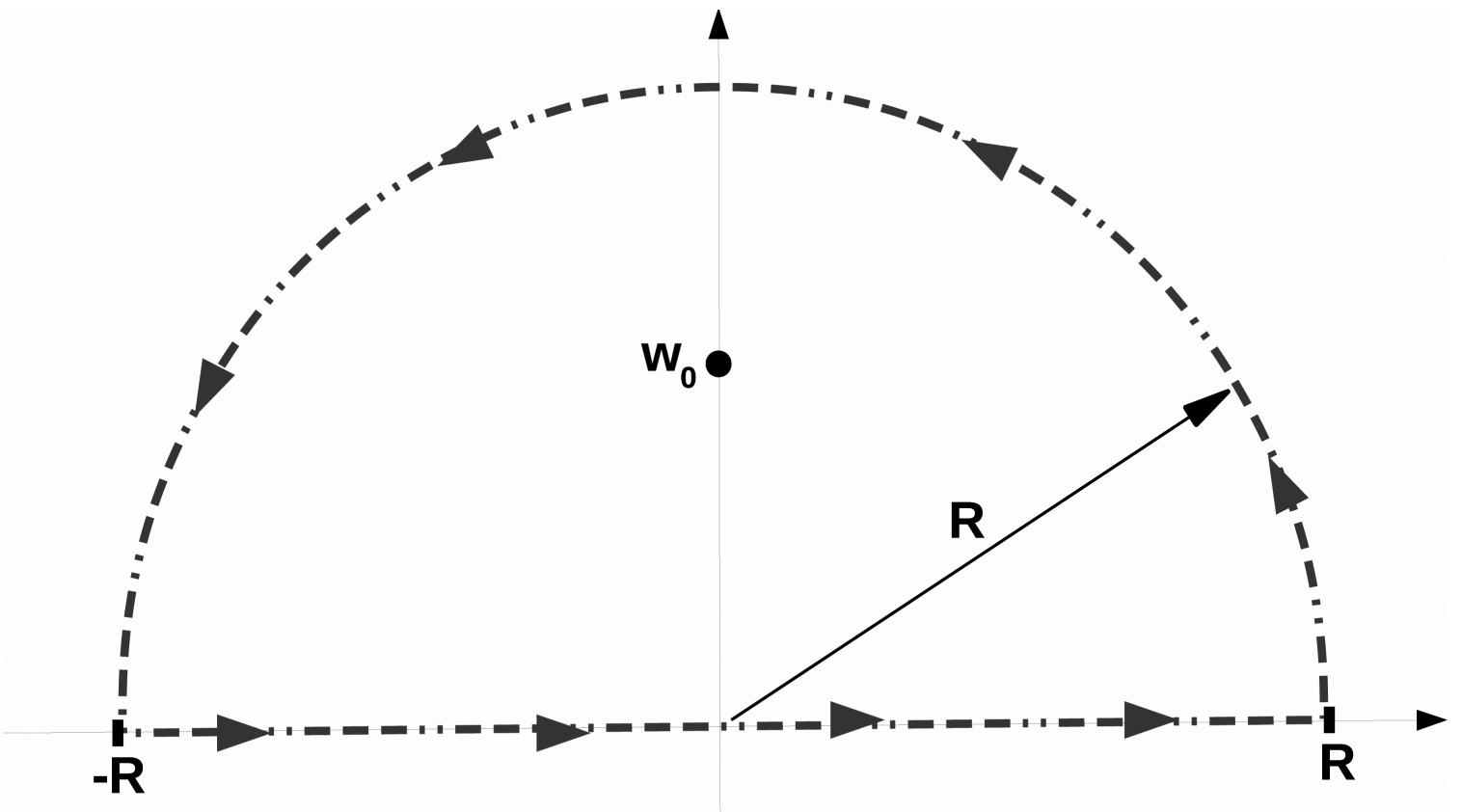

Figure 3: Path of the complex-plane integral from Eq. (47). The upper arch is the integral of $\left(w-w_{0}\right)^{-1}$ when $w=R e^{i \theta}$, with $\theta$ varying from 0 to $\pi$. The integral over the real line corresponds to integrating $\left(w-w_{0}\right)^{-1}$ from $-R$ to $R$, which in the limit when $R \rightarrow \infty$ is what we want to calculate. Together, these two add up to the circuit integral $\oint \mathrm{d} w\left(w-w_{0}\right)^{-1}$, which equals $2 \pi i$ times the residues inside the circuit, which for the single pole $w=w_{0}$, is 1 .

$$
\int_{-\infty}^{\infty} \mathrm{d} w \frac{1}{w-w_{0}}=2 i \pi-\lim _{R \rightarrow \infty} \int_{0}^{\pi} \mathrm{d} \theta \frac{i R e^{i \theta}}{R e^{i \theta}-w_{0}}=2 i \pi-i \int_{0}^{\pi} \mathrm{d} \theta=i \pi .
$$

If the student is not acquainted with residues, they can understand this result by integrating from $-R$ to $R$ and later taking the limit $R \rightarrow \infty$. The integral is obviously a logarithm:

$$
\int_{-\infty}^{\infty} \mathrm{d} w \frac{1}{w-w_{0}}=\lim _{R \rightarrow \infty} \int_{-R}^{R} \mathrm{~d} w \frac{1}{w-w_{0}}=\lim _{R \rightarrow \infty} \ln \left(\frac{R-w_{0}}{-R-w_{0}}\right)=\ln (-1) .
$$

As $e^{i \pi}=-1$, it is possible to see why the result of the integral is $i \pi$.

Replacing this result in Eq. (46), as well as the definition of $w_{0}$, we find

$$
\int_{0}^{\pi} \mathrm{d} \theta \frac{\varepsilon}{\varepsilon+\cos \theta}=\frac{\varepsilon}{\varepsilon-1} \frac{i \pi}{w_{0}}=\frac{\varepsilon \pi}{\sqrt{\varepsilon^{2}-1}} .
$$

Replacing this in Eq. (45) and then in Eq. 44) and in Eq. (43), we find the general result

$$
\int_{0}^{N \pi} \mathrm{d} \theta \frac{\sin ^{2} \theta}{(\varepsilon+\cos \theta)^{2}}=N \frac{\varepsilon \pi}{\sqrt{\varepsilon^{2}-1}}-N \pi
$$

Then, integral (40), from Sec. 3, becomes, with $N=1$, while for the integral (41), from Sec. 4, we have $N=2$,

$$
\int_{0}^{2 \pi} \mathrm{d} \theta \frac{\sin ^{2} \theta}{\left(\varepsilon^{-1}+\cos \theta\right)^{2}}=2 \pi\left\{\frac{1}{\sqrt{1-\varepsilon^{2}}}-1\right\}
$$




\section{Acknowledgements}

L. A. C. acknowledges support from the Coordenação de Aperfeiçoamento de Pessoal de Nível Superior (CAPES). C. A. B. thanks Karla Pelogia (Fakultät 9/Universität Stuttgart), Thaís Victa Trevisan (IFGW/UNICAMP) and Amit Hagar (HPSM/Indiana University Bloomington) for their help in locating some of the references used in this paper. R. d. J. N. thanks Lia M. B. Napolitano for the valuable discussions during the calculations.

\section{References}

[1] Robert Eisberg and Robert Resnick, Quantum Physics of Atoms, Molecules, Solids, Nuclei, and Particles (John Wiley \& Sons, New York, 1985).

[2] Robert Eisberg and Robert Resnick, Fundamentals of Modern Physics (John Wiley \& Sons, New York, 1961).

[3] Sin-Itiro Tomonaga, Quantum Mechanics (NorthHolland Publishing Company, Amsterdam, 1968), v. 1.

[4] David Joseph Bohm, Quantum Theory (Dover Publications Inc., New York, 1979).

[5] Max Born, Atomic Physics (Blackie \& Son Ltd., London, 1937).

[6] Arnold Sommerfeld, Atomic Structure and Spectral Lines (Methuen \& Co. Inc., London, 1923).

[7] William Wilson, Philosophical Magazine 29, 795 (1915).

[8] Bartel Leendert van der Waerden, Sources of Quantum Mechanics (Dover Publications Inc., Mineola, 2007).

[9] Jun Ishiwara, Proceedings of the Tokyo Mathematico-Physical Society 8, 106 (1915). English translation in progress by Karla Pelogia and Carlos Alexandre Brasil.

[10] Arnold Sommerfeld, Annalen der Physik 51, 1 (1916).

[11] Arnold Sommerfeld, Annalen der Physik 51, 125 (1916).

[12] Claude Cohen-Tannoudji, Bernard Diu, and Franck Laloë, Quantum Mechanics (John Wiley \& Sons, New York, 1977), vol. 2.

[13] Jun John Sakurai, Advanced Quantum Mechanics (Addison-Wesley, Massachusetts, 1967).

[14] Nelson Studart, Revista Brasileira de Ensino de Física 22, 523 (2000).

[15] Max Jammer, The Conceptual Development of Quantum Mechanics (McGraw-Hill Book Company, New York, 1966).

[16] Among those, deserves mention the paper Werner Heisenberg published in the Zeitschrift für Physik in 1925. Its English translation, Quatum-Theoretical
Re-Interpretation of Kinematic and Mechanical Relations can be found at Ref. [8] and at Ref. [67].

[17] Erwin Schrödinger's 1926 works published in the Annalen der Physik are of particular importance, and their English translations, Quantisation as a problem of proper values parts I-IV, can be found at Erwin Schrödinger, Collected Papers on Wave Mechanics (Chelsea Publishing Company, New York, 1978) and at Ref [67].

[18] Manfred Bucher, arXiv:0802.1366 (2008).

[19] Thomas S. Kuhn, Black-Body Theory and Quantum Discontinuity 1894-1912 (Claredon Press, Oxford, 1978).

[20] Norbert Straumann, European Physical Journal H 36, 379 (2011).

[21] Bruno Feldens, Penha Maria Cardoso Dias e Wilma Machado Soares Santos, Revista Brasileira de Ensino de Física 32, 2602 (2010).

[22] D. ter Haar, The Old Quantum Theory (Pergamon Press Ltd., Oxford, 1967).

[23] Max Planck, Revista Brasileira de Ensino de Física 22, 536 (2000).

[24] Max Planck, Revista Brasileira de Ensino de Física 22, 538 (2000).

[25] Niels Bohr, Philosophical Magazine 26, 1 (1913).

[26] Niels Bohr, Philosophical Magazine 26, 476 (1913).

[27] Niels Bohr, Philosophical Magazine 26, 857 (1913).

[28] Niels Bohr, Textos Fundamentais da Física Moderna - Sobre a Constituição de Átomos e Moléculas (Fundação Calouste Gulbenkian, Lisbon, 1963).

[29] F.A.G. Parente, A.C.F. dos Santos and A.C. Tort, Revista Brasileira de Ensino de Física 35, 4301 (2013).

[30] Helge Kragh, Physics Today 66, 36 (2013).

[31] Anatoly Svidzinsky, Marlan Scully and Dudley Herschbach, Physics Today 67, 33 (2014).

[32] Leo Banet, American Journal of Physics 34, 496 (1966).

[33] Leo Banet, American Journal of Physics 38, 821 (1970).

[34] Arnold Sommerfeld, European Physical Journal H 39, 157 (2014).

[35] Arnold Sommerfeld, European Physical Journal H 39, 179 (2014).

[36] Helge Kragh, European Physical Journal H 36, 327 (2011).

[37] Oliver Darrigol, Studies in History and Philosophy of Science 40, 151 (2009).

[38] http://www.nobelprize.org/nobel_prizes/ physics/laureates/1922/, accessed on September, 11th, 2015.

[39] Richard L. Liboff Physics Today 37, 50 (1984).

[40] Edmund Whittaker, A History of the Theories of Aether and Electricity (Thomas Nelson \& Sons, London, 1953).

[41] Adam J. Makowski, European Journal of Physics 27, 1133 (2006). 
[42] According to Ref. [20], Planck chose the letter $h$ exactly because this was usually used as the variable whose limit was taken to zero $\lim _{h \rightarrow 0}$ in calculus textbooks.

[43] Ghazi Q. Hassoun and Donald H. Kobe, American Journal Physicas 57, 658 (1989).

[44] Bibhas Bhattacharyya, European Journal of Physics 27, 497 (2006).

[45] Michael Eckert, European Physical Journal H 39, 141 (2014).

[46] Alexander L. Fetter and John Dirk Walecka, Theoretical Mechanics of Particles and Continua (Dover, Mineola, 2003).

[47] These articles by Sommerfeld in Ref. [10-11] have no accessible English translations, even though his ideas are found in his book mentioned in Ref. [6] and in the translations of his previous communications to the Bavarian Academy of Sciences in Ref. [34] and Ref. [35].

[48] The original article was published in the Mémoires de l'Académie Royale des Sciences et des Lettres de Danemark, an English translation of which, On the Quantum Theory of Line-Spectra, can be found in the book mentioned in Ref. [8].

[49] The $f$ was at times replaced by a $b$, for Bergman.

[50] Suman Seth, Studies in History and Philosophy of Science 39, 335 (2008).

[51] Suman Seth, Studies in History and Philosophy of Science 40, 303 (2009).

[52] http://www.nobelprize.org/nobel_prizes/ physics/laureates/1945/pauli-lecture.pdf, accessed on September, 15th, 2015.

[53] If one prefers not to employ this approximation, use the reduced mass of the system $\mu$ instead of the mass of the electron $m$.

[54] Here we used Gaussian units, so no cumbersome constants have to be carried in the calculations. If the student is more used to SI units, they may replace $Z$ with $Z /\left(4 \pi \epsilon_{0}\right)$.

[55] Helge Kragh, European Journal of Physics 24, 169 (2003).
[56] Helge Kragh, Archive for History of the Exact Sciences 57, 395 (2003).

[57] Helge Kragh, Historical Studies in the Physical Sciences 15, 67 (1985).

[58] Andreas Terzis, European Journal of Physics 29, 735 (2008).

[59] http://www.nobelprize.org/nobel_prizes/ physics/laureates/1918/planck-lecture. html, accessed on September 17th, 2015.

[60] Sergio DeBenedetti, C.E. Cowan, Wilfred R. Konneker and Henry Primakoff, Physical Review 77, 205 (1950).

[61] Ana Carolina Bruno Machado, Vicente Pleitez and M.C. Tijero, Revista Brasileira de Ensino de Física 28, 407 (2006).

[62] Anthony Duncan and Michel Janssen, Studies in History and Philosophy of Science 48, 68 (2014).

[63] Anthony Duncan and Michel Janssen, arXiv:1404.5341

[64] Refs. [62-63] present not just a little of the history, but also the complete original calculations of the Stark effect and comparison with modern methods.

[65] Edward MacKinnon, American Journal of Physics 44, 1047 (1976).

[66] Louis de Broglie and Léon Brillouin, Selected Papers on Wave Mechanics (Blackie \& Son Limited, London, 1929).

[67] Gunter Ludwig, Wave Mechanics (Pergamon Press, Oxford, 1968).

[68] Lawrence Christian Biedenharn, Foundations of Physics 13, 13 (1983).

[69] Peter Vickers, European Journal for Philosophy of Science 2, 1 (2012).

[70] Arnold Sommerfeld, American Journal of Physics 17, 315 (1949).

[71] Max Born, Mechanics of the Atom (Bell, London, 1927).

[72] George Brown Arfken, Mathematical Methods for Physicists (Academic Press, San Diego, 1985), 3rd ed.

[73] Eugene Butkov, Mathematical Physics (AddisonWesley, Reading, 1968). 